\title{
Is the Screening Tool for the Assessment of Malnutrition in Paediatrics (STAMP) enough to fulfil the Care Quality Commission (CQC) Outcome 5 'Meeting Nutritional Needs' criteria for nutritional screening in a tertiary paediatric centre?
}

\author{
L. Cooke, S. Trace, H. Morris and T. Cimonetti \\ Department of Nutrition and Dietetics, Bristol Royal Hospital for Children, Upper Maudlin Street, Bristol BS2 8BJ, UK
}

The Care Quality Commission (CQC) launched in April $2010^{(1)}$ supports a quality of care outcomes framework for hospitals. Outcome 5 -'Meeting Nutritional Needs' expects everybody admitted to hospital to be 'nutritionally screened within 24 hours of admission'. Following the CQC launch, the Bristol Royal Hospital for Children (BRHC) dietetic department led the implementation of the STAMP validated paediatric nutrition screening tool ${ }^{(2)}$. Its suitability following implementation was evaluated.

After piloting the tool on Paediatric medical and surgical wards it was rolled out across the hospital population. Data was collected between November 2010 and March 2011 as part of the CQC data collection. The hospital registered this data collection through the hospital audit team on a monthly basis in order to record compliance status, reporting back to the trust CQC governance lead. All children admitted for longer than 24 hours aged between 0-18 years were included. STAMP is currently only validated for children aged $2-16$ years. STAMP only screened $56 \%$ of our patient population as on average the other $44 \%$ are less than 2 years of age. $17 \%$ of patients are already under the care of the dietetic team - i.e. those with a chronic illness who have ongoing dietetic care. In order to streamline the nursing nutritional screening process to quickly identify children above 2 years of age and those children who were not already under the ongoing care of a dietitian, a Nutritional Screening Flow Chart (NSFC) was developed. The NSFC also triggers the nursing staff to inform the dietetic service when patients with the following conditions are admitted: Metabolic disorders, Cystic Fibrosis, Diabetes, Critical Care, Renal Failure, Bone Marrow Transplant or needing Ketogenic diets, as they will already be under dietetic care.

In conclusion, STAMP is only useful to screen $39 \%$ of BRHC patients as the remaining $61 \%$ are either under the age of 2 or already part of an ongoing specialist dietetic caseload. STAMP, in isolation, is not a suitable screening tool for use across a paediatric tertiary inpatient establishment in order to fulfil the CQC requirements for Outcome 5. Alternative nutritional screening needs to be sought to identify malnutrition in under two's and prevent replication of screening patients already under the care of the hospital dietitians. The CQC should be made aware that there is currently no validated nurse-delivered screening tool that can identify malnutrition in $100 \%$ of children admitted to a tertiary paediatric hospital and need to adapt its outcome measures accordingly.

1. http://www.cqc.org.uk/_db/_documents/PCA_OUTCOME_5_new.doc.

2. McCarthy H, McNulty H, Dixon M \& Eaton-Evans MJ (2009) The validation of a nutritional screening tool for Children. Proceedings of the Nutrition society $\mathbf{6 8}(\mathrm{OCEI})$, E78. 